DOI: 10.17516/1997-1397-2021-14-5-659-666

УДК 517.95

\title{
On an Inverse Problem for a Stationary Equation with Boundary Condition of the Third Kind
}

\author{
Alexander V. Velisevich* \\ Siberian Federal University \\ Krasnoyarsk, Russian Federation
}

Received 10.04.2021, received in revised form 10.05.2021, accepted 20.06.2021

\begin{abstract}
The identification of an unknown coefficient in the lower term of elliptic second-order differential equation $M u+k u=f$ with boundary condition of the third kind is considered. The identification of the coefficient is based on integral boundary data. The local existence and uniqueness of the strong solution for the inverse problem is proved.
\end{abstract}

Keywords: inverse problem for PDE, boundary value problem, second-order elliptic equation, existence and uniqueness theorem.

Citation: A.V. Velisevich, On an Inverse Problem for a Stationary Equation with Boundary Condition of the Third Kind, J. Sib. Fed. Univ. Math. Phys., 2021, 14(5), 659-666.

DOI: 10.17516/1997-1397-2021-14-5-659-666.

\section{Introduction}

In this paper an inverse problem for some stationary equation is considered.

Problem. For given functions $f(x), \sigma(x), \beta(x), h(x)$ and constant $\mu$ find function $u(x)$ and constant $k$ that satisfy the equation

$$
-\operatorname{div}(\mathcal{M}(x) \nabla u)+m(x) u+k u=f
$$

boundary condition

$$
\left.\left(\frac{\partial u}{\partial \bar{N}}+\sigma(x) u\right)\right|_{\partial \Omega}=\beta(x)
$$

and the condition of overdetermination

$$
\int_{\partial \Omega} u h(x) d s=\mu \text {. }
$$

Here $\Omega \subset \mathbf{R}^{n}$ is a bounded domain with boundary $\partial \Omega, t \in(0, T), \mathcal{M}(x) \equiv\left(m_{i j}(x)\right)$ is a matrix of functions $m_{i j}(x), i, j=1,2, \ldots, n ; m(x)$ is a scalar function, $\frac{\partial}{\partial \bar{N}}=(\mathcal{M}(x) \nabla, \mathbf{n}), \mathbf{n}$ is the unit vector of the outward normal to the boundary $\partial \Omega$.

A main goal of this paper is to establish the existence and uniqueness of the strong solution of inverse problem (1)-(3). The additional integral boundary data similar to condition of overdetermination (3) were considered [1-3]. Following the idea given in [1-3] and using method

*velisevich94@mail.ru

(c) Siberian Federal University. All rights reserved 
developed in [4], we prove the existence of the solution by reducing the inverse problem to an operator equation of the second kind for the unknown coefficient. Note that the problem for the same equation with Dirichlet boundary conditions was considered [5].

The study of inverse problems for the elliptic equations goes back to fundamental work of M. M. Lavrentiev [6]. Inverse problems for the elliptic equation with special boundary conditions (non-local conditions, non-classical conditions) were considered [7-9].

Such problems arise in determination of unknown physical properties of a medium. In particular, the lowest coefficient $k$ specifies, for instance, the catabolism of contaminants due to chemical reactions [10] or the absorption in diffusion and acoustic problems [11].

\section{The preliminaries}

The following notations are used $\|\cdot\|_{R},(\cdot, \cdot)_{R}$ - the norm and the inner product in $\mathbb{R}^{n}$; $\|\cdot\|,(\cdot, \cdot)$ - the norm and the inner product in $L^{2}(\Omega) ;\|\cdot\|_{j},\langle\cdot, \cdot\rangle_{1}$ - the norm in $W_{2}^{j}(\Omega)$,

$j=1,2$, and the duality relation between $\stackrel{\circ}{W_{2}^{1}}(\Omega)$ and $W_{2}^{-1}(\Omega)$, respectively. The linear operator $M: W_{2}^{1}(\Omega) \rightarrow\left(W_{2}^{1}(\Omega)\right)^{*}$ of the form

$$
M=-\operatorname{div}(\mathcal{M}(x) \nabla)+m(x) I,
$$

Is introduced, where $I$ is the identity operator. The notation

$$
\left\langle M v_{1}, v_{2}\right\rangle_{M}=\int_{\Omega}\left(\left(\mathcal{M}(x) \nabla v_{1}, \nabla v_{2}\right)_{R}+m(x) v_{1} v_{2}\right) d x
$$

is also used for $v_{1}, v_{2} \in W_{2}^{1}(\Omega)$. The following assumptions hold throughout the paper

I. $m_{i j}(x), \partial m_{i j} / \partial x_{l}, i, j, l=1,2, \ldots, n$, and $m(x)$ are bounded in $\Omega$. Operator $M$ is elliptic, that is, there exist positive constants $m_{0}$ and $m_{1}$ such that for all $v \in W_{2}^{1}(\Omega)$

$$
m_{0}\|v\|_{1}^{2} \leqslant\langle M v, v\rangle_{M} \leqslant m_{1}\|v\|_{1}^{2}
$$

II. $M$ is self-adjoint, that is, $m_{i j}(x)=m_{j i}(x)$ for $i, j=1, \ldots, n$.

The existence and uniqueness results for problem (1)-(3) is based on two lemmas for direct problem (1)-(2) with known coefficient $k$.

Lemma 1.1. Let $u$ be the strong solution of problem (1)-(2). If $f \geqslant 0, \beta \geqslant 0, \sigma \geqslant 0, k>0$ and assumptions $I$, II are fulfilled, then $u \geqslant 0$ almost everywhere in $\Omega$.

Proof. Multiplying (1) by $\bar{u}=\min \{\bar{u}, 0\}$ in terms of the inner product in $L_{2}(\Omega)$ and integrating by parts in first term, we obtain

$$
\langle M \bar{u}, \bar{u}\rangle_{1}+k\|\bar{u}\|^{2}+\int_{\partial \Omega} \sigma \bar{u}^{2} d s-\int_{\partial \Omega} \beta \bar{u} d s-(f, \bar{u})=0 .
$$

Taking into account the lemma conditions, the last equality implies that

$$
m_{1}\|\bar{u}\|_{1}^{2} \leqslant 0
$$

So, $\bar{u}=0$ almost everywhere in $\Omega$. Lemma is proved. 
Lemma 1.2. Let $u_{1}, u_{2} \in W_{2}^{2}(\Omega)$ are the solutions of the problems

$$
\begin{gathered}
M u_{i}+k_{i} u_{i}=f_{i}, \\
\left.\left(\frac{\partial u_{i}}{\partial \bar{N}}+\sigma u_{i}\right)\right|_{\partial \Omega}=\beta_{i},
\end{gathered}
$$

here $i=1,2$.

If $0 \leqslant k_{1} \leqslant k_{2}, 0 \leqslant \beta_{2} \leqslant \beta_{1}, 0 \leqslant f_{2} \leqslant f_{1}$ and $\sigma(x) \geqslant 0$ then $u_{1} \geqslant u_{2} \geqslant 0$ for almost all $x \in \bar{\Omega}$.

Proof. By Lemma (1.1), $u_{i} \geqslant 0, i=1,2$, for almost all $x \in \bar{\Omega}$. The difference $u_{1}-u_{2}$ satisfies equation

$$
M\left(u_{1}-u_{2}\right)+k_{1}\left(u_{1}-u_{2}\right)=\left(k_{2}-k_{1}\right) u_{2}+f_{1}-f_{2},
$$

and boundary condition

$$
\left.\left(\frac{\partial\left(u_{1}-u_{2}\right)}{\partial \bar{N}}+\sigma(x)\left(u_{1}-u_{2}\right)\right)\right|_{\partial \Omega}=\beta_{1}-\beta_{2} .
$$

Taking into account the lemma conditions, the right side of (5) is non-negative and $\beta_{1}-\beta_{2} \geqslant 0$. So, by Lemma (1.1), $u_{1}-u_{2} \geqslant 0$ for almost all $x \in \bar{\Omega}$. Lemma is proved.

\section{Existence and uniqueness}

First of all the solution of the inverse problem should be defined. By the solution of the inverse problem is meant function $u \in W_{2}^{2}(\Omega)$ and a positive real number $k$. They satisfy equation (1) almost everywhere in $\Omega$ and conditions (2)-(3) almost everywhere on $\partial \Omega$. Now, to formulate the theorem functions $a, a^{\tau}$ and $b$ are introduced as the solution of the problems

$$
\begin{gathered}
M a=f(x),\left.\quad\left(\frac{\partial a}{\partial \bar{N}}+\sigma(x) a\right)\right|_{\partial \Omega}=\beta(x) ; \\
M a^{\tau}+\tau a^{\tau}=f,\left.\quad\left(\frac{\partial a^{\tau}}{\partial \bar{N}}+\sigma(x) a^{\tau}\right)\right|_{\partial \Omega}=\beta(x) ; \\
M b=0,\left.\quad\left(\frac{\partial b}{\partial \bar{N}}+\sigma(x) b\right)\right|_{\partial \Omega}=h(x),
\end{gathered}
$$

where $\tau>0$ is a real number.

Theorem 2.1. Let $\partial \Omega \in C^{2}$ and assumptions I, II are fulfilled. Suppose also that

(i) $f(x) \in L^{2}(\Omega), \beta(x), h(x) \in W_{2}^{3 / 2}(\partial \Omega), \quad \sigma(x) \in C(\partial \Omega)$;

(ii) $f(x) \geqslant 0$ almost everywhere in $\Omega ; \beta(x) \geqslant 0, \sigma(x) \geqslant 0, h(x) \geqslant 0$ for almost all $x \in \partial \Omega$ and there is a smooth piece $\Gamma$ of the boundary $\partial \Omega$ and a constant $\delta>0$ such that $\beta \geqslant \delta$ and $\omega \geqslant \delta$ almost everywhere on $\Gamma$.

Then problem (1)-(3) has a solution $\{u, k\}$. Moreover, the estimates

$$
a^{\tau} \leqslant u \leqslant a, \quad 0 \leqslant k \leqslant \tau, \quad\|u\|_{2} \leqslant C(\tau+1)\|a\|+\|a\|_{2}
$$

hold with some $\tau>0$, and constant $C$ depends on mes $\Omega, \tau, m_{0}$ and $m_{1}$. If

$$
0 \leqslant \mu-\Psi \leqslant \frac{m_{0}(a, b)^{2}}{\|a\|\|b\|},
$$

where $\Psi=\int_{\partial \Omega} a h d s-(f, b)$, then the solution is unique. 
Proof. Following the idea given in [4] and the method developed in [1], the original problem is reduced to an equivalent inverse problem with a non-linear operator equation for $k$. It follows from (1)-(3) that function $w=a-u$ and the constant $k$ satisfy the following relations

$$
\begin{gathered}
M w+k w=k a \\
\left.\left(\frac{\partial w}{\partial \bar{N}}+\sigma(x) w\right)\right|_{\partial \Omega}=0 \\
\int_{\partial \Omega} w h d s=\int_{\partial \Omega} a h d s-\mu .
\end{gathered}
$$

Taking into account (8), (11) and (12), multiplying (9) by $b$ in terms of the inner product in $L_{2}(\Omega)$ and integrating by parts twice, we obtain

$$
k(u, b)=\int_{\partial \Omega} a h d s+(f, b)-\mu=\Psi-\mu .
$$

Let operator $A: R_{+} \rightarrow R$ maps every $y \in R_{+}$into the real number $A y$ by the rule

$$
A y=\frac{\Psi-\mu}{\left(u_{y}, b\right)}
$$

where $u_{y}$ is the solution of direct problem (1)-(2) with $y=k$. One can show that the original problem is solvable if and only if operator $A$ has a fixed point, i.e., the operator equation $A k=k$ has a solution.

Now we need to prove that there exists $\tau>0$ such that operator $A$ defined for all $k \in[0, \tau]$, is continuous on $[0, \tau]$, and maps $[0, \tau]$ into itself. Indeed, Lemma 1.2 implies that for all $0 \leqslant y \leqslant \tau$

$$
a^{\tau} \leqslant u_{y} \leqslant a
$$

Therefore

$$
A y \geqslant \frac{\Psi-\mu}{(a, b)} \geqslant 0 .
$$

On the other hand, let us introduce the difference between (6) and (7)

$$
M\left(a-a^{\tau}\right)+\tau\left(a-a^{\tau}\right)=\tau a .
$$

Then, multiplying the difference by $a-a^{\tau}$ in terms of the inner product in $L^{2}(\Omega)$, integrating by parts in the first term and estimating the left-hand side of the result with the help of (4), we obtain

$$
m_{0}\left\|a-a^{\tau}\right\|_{1}^{2}+2 \int_{\partial \Omega} \sigma(a-a \tau)^{2} d s+2 \tau\left\|a-a^{\tau}\right\|^{2} \leqslant \frac{\tau^{2}}{m_{0}}\|a\|^{2} .
$$

This estimate and (15) allows one to obtain the lower bound of $\left(u_{y}, b\right)$ in $(14)$

$$
\left(u_{y}, b\right) \geqslant\left(a^{\tau}, b\right)=(a, b)-\left(a-a^{\tau}, b\right) \geqslant(a, b)-\frac{\tau}{\sqrt{m_{0}}}\|a\|\|b\| \geqslant 0 .
$$

Hence

$$
0 \leqslant \tau \leqslant \frac{\sqrt{m_{0}}(a, b)}{\|a\|\|b\|}
$$

In view of (14) and (16)

$$
A y \leqslant \frac{\Psi-\mu}{(a, b)-\frac{\tau}{\sqrt{m}_{0}}\|a\|\|b\|} \leqslant \tau
$$


Accordingly, the relation $A y \leqslant \tau$ holds for all $\tau>0$ such that

$$
\frac{\tau^{2}}{\sqrt{m_{0}}}\|a\|\|b\|-\tau(a, b)+\Psi-\mu \leqslant 0 .
$$

The last inequality is possible, because it follows from the theorem conditions that

$$
D \equiv(a, b)^{2}-\frac{4(\Psi-\mu)\|a\|\|b\|}{\sqrt{m_{0}}} \geqslant 0 .
$$

Then (17) is valid for $\tau$ that obeys the inequality

$$
\frac{\sqrt{m_{0}}((a, b)-\sqrt{D})}{2\|a\|\|b\|} \leqslant \tau \leqslant \frac{\sqrt{m_{0}}((a, b)+\sqrt{D})}{2\|a\|\|b\|} .
$$

Thus, the operator $A$ maps the segment into itself.

Now one can obtain the estimate of $u_{y}$ in $W_{2}^{1}(\Omega)$ provided that $y \in[0, \tau]$. Let $w_{y}=a-u_{y}$. This function satisfies (11)-(13) with $y=k$. Multiplying (11) for $k=y$ by $w_{y}$ in terms of the inner product in $L_{2}(\Omega)$ and integrating by parts in the first term, we obtain

$$
\left(M w_{y}, w_{y}\right)+y\left\|w_{y}\right\|^{2}=\left\langle M w_{y}, w_{y}\right\rangle_{M}+y\left\|w_{y}\right\|^{2}+\int_{\partial \Omega} \sigma w_{y}^{2} d s=y\left(a, w_{y}\right) .
$$

In view of (15) and the definition of $w_{y}$, we have

$$
\left|y \int_{\Omega} a w_{y} d x\right| \leqslant \tau\|a\|^{2} .
$$

Taking into account the ellipticity of operator $M$, the last two relations implies that

$$
\left\|u_{y}\right\|_{1} \leqslant \sqrt{\frac{\tau}{m_{0}}}\|a\|+\|a\|_{1} .
$$

In accordance with [12], direct problem (11)-(12) has a unique solution $w_{y} \in W_{2}^{2}(\Omega)$ for all $y \geqslant 0$. Furthermore, (11) is fulfilled almost everywhere in $\Omega$ and $M w_{y} \in L_{2}(\Omega)$. Multiplying (11) with $k=y$ by $M w_{y}$ in terms of the inner product in $L_{2}(\Omega)$ and integrating by parts in the second component, one can obtain the equality

$$
\left\|M w_{y}\right\|^{2}+y\left\langle w_{y}, M w_{y}\right\rangle_{M}+\int_{\partial \Omega} \sigma w_{y}^{2} d s=y\left(a M, w_{y}\right) .
$$

In accordance with (4), the second term of (19) is non-negative and

$$
y\left|\left(a, M w_{y}\right)\right| \leqslant \tau\|a\|\left\|M w_{y}\right\| \leqslant \frac{1}{2} \tau^{2}\|a\|^{2}+\frac{1}{2}\left\|M w_{y}\right\|^{2} .
$$

it follows from the last two relations that

$$
\left\|M w_{y}\right\|^{2} \leqslant \tau^{2}\|a\|^{2} .
$$

In view of the definition of $w_{y}$ and the inequality [12]

$$
\|v\|_{2} \leqslant C_{M}(\|M v\|+\|v\|),
$$


valid for all $v \in \stackrel{\circ}{W}_{2}^{1}(\Omega) \cap W_{2}^{2}(\Omega)$ with the constant $C_{M}$ depending on $M$ and mes $\Omega$, relations (18), (20) imply the estimate

$$
\|u\|_{2} \leqslant\left\|w_{y}\right\|_{2}+\|a\|_{2} \leqslant C_{M}(\tau+1)\|a\|+\|a\|_{2} .
$$

Now one can show that operator $A$ is continuous on segment $[0, \tau]$. Let $y_{1}, y_{2} \in[0, \tau]$ and $u_{y_{1}}, u_{y_{1}}$ are the solutions of problem (11), (12) with $y_{1}=k$ and $y_{2}=k$, respectively. By the definition of operator $A,(15)$ and (16)

$$
\left|A_{y_{1}}-A_{y_{2}}\right| \leqslant \frac{\left\|u_{y_{2}}-u_{y_{1}}\right\|\|b\|(\Psi-\mu)}{\left(a^{\tau}, b\right)^{2}} \leqslant \frac{\left\|u_{y_{2}}-u_{y_{1}}\right\|\|b\|(\Psi-\mu)}{\left((a, b)-\frac{\tau}{\sqrt{m_{1}}}\|a\|\|b\|\right)^{2}} .
$$

On the other hand, multiplying the difference of equation (1) for $k=y_{1}$ and $k=y_{2}$ by $u_{y_{1}}-u_{y_{2}}$ in terms of the inner product in $L_{2}(\Omega)$ and integrating by parts in the first term of the resulting equality, we obtain

$$
\left\langle M\left(u_{y_{1}}-u_{y_{2}}, u_{y_{1}}\right)\right\rangle_{1}+\int_{\partial \Omega} \sigma\left(u_{y_{1}}-u_{y_{2}}\right)^{2} d s+y_{1}\left\|u_{y_{1}}-u_{y_{2}}\right\|^{2}=\left(y_{2}-y_{1}\right)\left(u_{y_{2}}, u_{y_{1}}-u_{y_{2}}\right) .
$$

In accordance with (4) and the non-negativity of $y_{1}$, the left side of (22) can be estimated as

$$
\left\langle M\left(u_{y_{1}}-u_{y_{2}}\right), u_{y_{1}}\right\rangle_{1}+\int_{\partial \Omega} \sigma\left(u_{y_{1}}-u_{y_{2}}\right)^{2} d s+y_{1}\left\|u_{y_{1}}-u_{y_{2}}\right\|^{2} \geqslant m_{0}\left\|u_{y_{1}}-u_{y_{2}}\right\|_{1}^{2} .
$$

The right term of (22) is estimated with the use of (15) as

$$
\left|\left(y_{2}-y_{1}\right)\left(u_{y_{2}}, u_{y_{1}}-u_{y_{2}}\right)\right| \leqslant \frac{1}{2 m_{0}}\left|y_{2}-y_{1}\right|^{2}\|a\|^{2}+\frac{m_{0}}{2}\left\|u_{y_{1}}-u_{y_{2}}\right\|_{1}^{2} .
$$

Hence, we obtain the relation

$$
\left\|u_{y_{1}}-u_{y_{2}}\right\|_{1} \leqslant \frac{1}{m_{0}}\|a\|\left|y_{2}-y_{1}\right| .
$$

Then, joining (21) with

$$
\tau=\frac{2 \sqrt{m_{0}}((a, b)-\sqrt{D})}{\|a\|\|b\|}=\tau_{0}
$$

and (23), we obtain the inequality

$$
\left|A y_{1}-A y_{2}\right| \leqslant \frac{\|a\|\|b\|(\Psi-\mu)}{((a, b)+2 \sqrt{D})^{2}}\left|y_{1}-y_{2}\right|
$$

which implies the continuity of operator $A$. Thus, according to the Brouwer fixed point theorem, operator $A$ has a fixed point $k^{*} \in\left[0, \tau_{0}\right]$ and the pair $\left\{u^{*}, k^{*}\right\}$, where function $u^{*}$ satisfies (1)-(2) with $k=k^{*}$, gives a solution of problem (1)-(3).

It remains to prove that the solution of problem (1)-(3) is unique under assumption (10). In this case, operator $A$ is a contractor on the segment $\left[0, \tau_{0}\right]$ because $A$ satisfies (24) with

$$
q=\frac{\|a\|\|b\|(\Psi-\mu)}{(a, b)+2 \sqrt{D}}<\frac{(a, b)^{2}}{((a, b)+2 \sqrt{D})^{2}}<1 .
$$

Let us assume that $\left(u^{\prime}, k^{\prime}\right)$ and $\left(u^{\prime \prime}, k^{\prime \prime}\right)$ are two solutions of problem (1)-(2). Then $k^{\prime}, k^{\prime \prime}$ are the fixed points of operator $A$. By (24)

$$
\left|k^{\prime}-k^{\prime \prime}\right|=\left|A k^{\prime}-A k^{\prime \prime}\right| \leqslant q\left|k^{\prime}-k^{\prime \prime}\right|
$$


whence $k^{\prime}-k^{\prime \prime}=0$. This in turn implies $u^{\prime}-u^{\prime \prime}=0$ in view of (23). Theorem is proved.

Under assumption (10) the solution $\{u, k\}$ depends continuously on the input data of original problem.

Remark 1. Condition (4) is valid when $m(x) \geqslant m_{0}>0$ almost everywhere in $\Omega$, or $\sigma(x) \geqslant \sigma_{0}>0$ almost everywhere in $\partial \Omega$, here $m_{0}, \sigma_{0}$ are some constants. In the last case left inequality holds due to the Friedrichs inequality.

Remark 2. The main theorem is correct for a more general type of operator $M$ :

$$
M=-\operatorname{div}(\mathcal{M}(x) \nabla)+(\bar{m} \nabla)+m(x) I,
$$

where $\bar{m} \in L_{\infty}(\Omega)$ is vector of functions $m_{i}(x), i=1, \ldots, n$.

This work was supported by Russian Foundation of Basic Research [grant no. 20-31-90053].

\section{References}

[1] A.Sh.Lyubanova, Identification of a constant coefficient in an elliptic equation, Applicable Analysis, 87(2008), 1121-1128. DOI: 10.1080/00036810802189654

[2] A.Sh.Lyubanova, A.Tani, An inverse problem for pseudoparabolic equation of filtration: the existence, uniqueness and regularity, Applicable Analysis, 90(2011), 1557-1571.

DOI: $10.1080 / 00036811.2010 .530258$

[3] A.Sh.Lyubanova On an inverse problem for quasi-linear elliptic equation, J. Sib. Fed. Univ. Math. Phys., 8(2015), 38-48.

[4] A.I.Prilepko, D.G.Orlovsky, I.A.Vasin, Methods for solving inverse problems in mathematical physics, New York: Marcel Dekker, 2000.

[5] A.Sh.Lyubanova, A.V.Velisevich, Inverse problems for the stationary and pseudoparabolic equations of diffusion, Applicable Analysis, 98(2019), 1997-2010.

DOI: $10.1080 / 00036811.2018 .1442001$

[6] M.M.Lavrentiev, On some inverse ill-posed problems of mathematical physics, Novosibirsk, Nauka, 1962 (in Russian).

[7] K.B.Sabitov, N.V.Martem'yanova, An inverse problem for an equation of elliptic-hyperbolic type with a non-local boundary condition, Siberian Math. J., 53(2012), 507-519.

[8] F.Kanca, Inverse coefficient problem for a second?order elliptic equation with nonlocal boundary conditions, Mathematical Methods in Applied Science, 39(11)(2015), 3152-3158.

[9] R.Amirov, Y.Mehraliyev, N.Heydarzade, On an inverse boundary-value problem for a second-order elliptic equation with non-classical boundary conditions, Cumhuriet Science Journal, 41(2)(2020), 443-455. DOI: 10.17776/csj.684366

[10] G.V.Alekseev, E.A.Kalinina, Identification of the lowest coefficient of a stationary convention-diffusion-reaction equation, Sib. Zh. Ind. Mat., 10(2007), 3-16 (in Russian).

[11] H.Egger, J-F.Pietschmann, M.Schlobottom, Simultaneous identification of diffusion and absorption coefficients in a quasi-linear elliptic problem, Inverse Problems, 30(2014), 035009. DOI: $10.1088 / 0266-5611 / 30 / 3 / 035009$ 
[12] O.A.Ladyzhenskaya, N.N.Uralceva, Linear and quasilinear elliptic equations, New York: Academic Press, 1973, (English transl. Moskva, Nauka, 1964).

\title{
Об одной обратной задаче для эллиптического уравнения со смешанными граничными условиями третьего рода
}

\section{Александр В. Велисевич}

\begin{abstract}
Аннотация. В данной работе рассматривается обратная задача для эллиптического уравнения с граничными условием третьего рода и условием интегрального переопределения. Доказано существование и единственность решения, а также непрерывная зависимость решения от входных данных.
\end{abstract}

Ключевые слова: обратная задача, краевая задача, эллиптическое уравнение, теорема существования и единственности. 